NASZA DERMATOLOGIA Online OUR DERMATOLOGY Online

Source of Support: Nil

Competing Interests: None

\section{A SURVEY OF NAIL INFECTION AND AWARENESS AMONG NON-DIABETIC PATIENTS IN MAURITIUS}

\author{
Jeetun Oomar, Jeewon Rajesh \\ Department of Health Sciences, Faculty of Science, Reduit, University of Mauritius, \\ Mauritius
}

Corresponding author: Dr Jeewon Rajesh $\mathrm{PhD}$.

r.jeewon@uom.ac.mu

\begin{abstract}
Introduction: Nail infection like onychomycosis is mainly caused by dermatophytes and account for almost half of all nail disorders. Prevalence of nail infection has been attributed to several factors such as age, gender, socioeconomic status and predisposition to diabetes amongst others. This study aims at determining the prevalence and level of awareness of non-diabetics towards nail infections in Mauritius. Material and Methods: A survey was carried out among 471 participants of the non-diabetic population of Mauritius. Data on socio demographic factors, awareness, level of hygiene, family history and quality of life were obtained via questionnaire based studies. Data was analysed using the SPSS software.

Results: Results show almost the same ratio of female to male was affected with nail infection but varies gender wise. Participants within the age group 20-60 with less than US 500 monthly income had a higher incidence of nail infection. No significant relation was obtained between nail infection and education level. A significant relationship was obtained between nail infection and occupation as well as quality of life. More than half of participants did not know about the routes of nail infections or the precautions needed to avoid spreading. However, good level of hygiene was observed among the participants.

Conclusion: Factors like age, gender and socio economic status had a significant relationship on nail infection. Nail infection affects the Quality of Life (QoL) and manual workers or even professionals are prone to nail infections.
\end{abstract}

Key words: non-diabetic; nail infection; awareness; socio demographic factors; quality of life

Cite this article:

Jeetun Oomar, Jeewon Rajesh: A survey of nail infection and awareness among non-diabetic patients in Mauritius. Our Dermatol Online. 2013; 4(3): 265-271

\section{Introduction}

There are several types of nail infections among which onychomycosis and Tinea unguium are more common [1]. Both fingernails and toenails are vulnerable to the infection. According to previous reviews, Onychomycosis is the most common nail disease which represents about $50 \%$ of all nail changes and for about $30 \%$ of all cutaneous fungal infections [2-6]. Nail infections usually exhibits itself as discoloration and thickening of the nail and crumbling edges. The latter manifest itself mostly in toenails $[5,6]$. Nail infection like onychomycosis is responsible for almost half of all nail disorders and one third of cutaneous fungal infections [5,7]. Research data in the United States have showed a prevalence of $18.5 \%$ [8], with the number of persons affected taking an upward trend [9]. Nail infections affect $32 \%$ of people between ages 60 and 70 and other studies conducted reported that $48 \%$ of the population may be affected by age 70 [10]. Toenail infections are several times more common than fingernail infections and are more difficult to treat because the toenails grow more slowly $[6,11]$.

It has been shown that the increasing incidence of diabetes, HIV infection, changes in lifestyle like an increase in urbanisation, the use of communal bathing facilities and footwear are the main factors leading in an increase in the prevalence of Tinea pedis and onychomycosis $[5,12]$. In rural Africa, Tinea pedis is uncommon, which is perhaps a reflection of a lack of associated risk factors such as the use of occlusive footwear and associated recreational activities [13]. Comparative study between nondiabetic and diabetic patients with nail changes showed that the latter had a higher proportion of onychomycosis relative to non-fungal onychodystrophy. It was also found that the nature of the fungal pathogens, dermatophytes prevailed mostly over yeast and non-dermatophytic moulds, both in diabetic and nondiabetic patients [14].

It has been shown that there are several important factors that are associated with the occurrence of nail infection such as age and gender [5]. A study of onychomycosis conducted among patients visiting physicians' offices found that the occurrence of onychomycosis considerably increased with age. For example, the prevalence of fungal nail infections was $0.7 \%$ in patients younger than 19 years of age, compared with $18.2 \%$ in patients 60- to 79-years of age. 
As far as gender is concerned and based on several studies, it has been observed that men are more prone to nail infections than women while women are more prone to candida onychomycosis than men [15]. Another important factor is the association of socio economic status (SES) on prevalence of nail infection. The prevalence of Tinea pedis was found to be higher among low SES than compared to high SES and similar results were obtained for prevalence of onychomycosis [16].

Knowledge and level of awareness of nail infection is quite important in either prevention or treatment of nail infection. A survey conducted in Ireland revealed that $84 \%$ of participants lack awareness about nail fungal infections and $40 \%$ of participants reported to podiatrists when the condition is at a severe to chronic stage [17]. Patients having nail infections had varying degrees of physical impairment and were limited both psychologically and socially thus resulting in a decrease of Quality of Life (QoL). They stated that women generally had a lower QoL compared to men ( $83 \%$ vs. $71 \%$ ). However patients satisfied with their treatment had an improvement in QoL [18]. To date, there are no documented studies on nail infection and awareness among non-diabetic patients in Mauritius. However a study on onychomycosis in a random sample of Mauritian diabetic patients found that $80 \%$ of dermatophyte isolated was Trichophyton species. They also found out that male patients were four times more likely to have nail infection than female patients [19]. Another survey of onychomycosis among 75 agricultural workers found that Trichophyton tonsurans was the most frequent fungus isolated in toe nails while no Candida infection was observed [20].

In this survey, an attempt has been made to determine the prevalence and level of awareness of non-diabetics towards nail infections. The main objectives are as follows:

1. To assess if there is any relationship between the severity of nail infection and demographic factors such as age and sex.

2. To determine the embarrassment and discomfort associated with nail infection and how quality of life is affected.

3. To identify whether there is a link between patient's occupation and nail infection.

4. To assess the level of awareness of participants towards nail infection.

\section{Material and Methods}

The study needed clearance from the UoM Research Ethics Committee and information sheet and consent form were distributed to participants willing to take part in the study.

Two questionnaires were used to obtain information from respondents. One was based mainly on:

a) Demographic information (age, gender, income, level of education, occupational status);

b) Podiatric details (hygiene, difficulties infected participants faced due to their nail infection);

c) Awareness (level of awareness of participants pertaining to nail infection).

The other one was based on standardised and validated question on Quality of life (psychological and physical difficulties faced by nail infected participants base on criteria set by DLQI [21]). The data collection was performed among members of the general population based on inclusion (general non-diabetic population of both genders of aged between 10 to 70 years old) and exclusion criteria (all diabetic people were excluded from the study while people with genetic nail disease like psoriasis were not considered).

\section{Data analysis:}

Statistical analysis of the data collected was done using the Statistical Package for Social Scientist, SPSS version 16.0 and Microsoft Excel.

\section{Results}

\section{Demographic details associated with participants}

Among the 471 participants, $45.6 \%$ of participants were male while $54.4 \%$ were female and out of which $15 \%$ had nail infections and $85 \%$ were not infected. More female (60.2\%) were recorded in the group age of 20-30 while higher frequency of male $(51.4 \%)$ were recorded in the group age of 31-60.

$17.2 \%$ of participants who had nail infections were male while $14.1 \%$ of participants having nail infections were female. The data was not found to be statistically significant $(p=0.336, r$ $=0.044$ ). Participants in the 20-60 age group were found to have a higher rate of nail infection as shown in Figure 1 but this was not found to be statistically significant ( $p=0.782, r=-0.013$ ). Male having had a tertiary level of education had a higher prevalence of nail infection $(44.2 \%)$. On the other hand, female having a secondary and tertiary level of education had an equal percentage of nail infection (38.3\%). Male having a salary of less than US \$ 500 had a higher prevalence of nail infection $(38.5 \%)$. However, female having no education at all had a higher percentage of nail infection $(50.0 \%)$. Participants of both gender having a job had higher prevalence of nail infection. $36 \%$ of participants having nail infection worked as manual workers while $28 \%$ were police officer.

Male living in humid regions was more prone to nail infection (43.6\%). However, female in semi- humid region had a higher rate of nail infection $(78.9 \%)$ but this was not significantly different.

\section{Podiatric details associated with participants}

Figure 2 shows that more participants answered that they sometime sweat $(62.3 \%)$ and rarely came into contact with chemical (51.9\%). Same trend were observed for wearing of gloves at work $(59.7 \%)$ but this was not statistically significant $(\mathrm{p}=0.004, \mathrm{r}=0.176)$.

More infected participants had their face that sweat the most $(37.1 \%)$. Male mostly experienced the hardening of the nail $(5.1 \%)$ while female a higher prevalence of nail discoloration $(5.1 \%)$. This was statistically significant $(\mathrm{p}=0.000, \mathrm{r}=$ -0.853). In addition, participants of both male and female did not know how they acquired the nail infection they suffered (35.9\% and $65.8 \%$ respectively). $41 \%$ of infected participants did not practice any hobbies while $30 \%$ of them regularly do gymnasium's sports. The prevalence of having an affected fingernail was low in both male and female $(9.3 \%$ and $8.2 \%$ respectively) while in male and female, one infected toenail had a higher prevalence that is $7 \%$ and $6.6 \%$ respectively.

Statistical analysis reveals a positive correlation between participant having nail infection and any other medical problem suffered suggesting that as participants having medical problem relating to nail infection, they were more prone to nail infection. A positive correlation was obtained when sweating and the approximate number of hours participants wear shoes per day were analysed $(r=0.067 ; p=0.004)$. 
Although all participants were non-diabetics, it is found that $45 \%$ of infected participants had member of their family suffering from diabetes while $14 \%$ had family members having nail infection. Both gender had a higher percentage of response for which they did not felt any discomforting symptoms. Male had a little difficulty to cut their nail compared to female. However more female participants found a little difficulty in wearing shoes that male. All these data were found to be statistically significant.

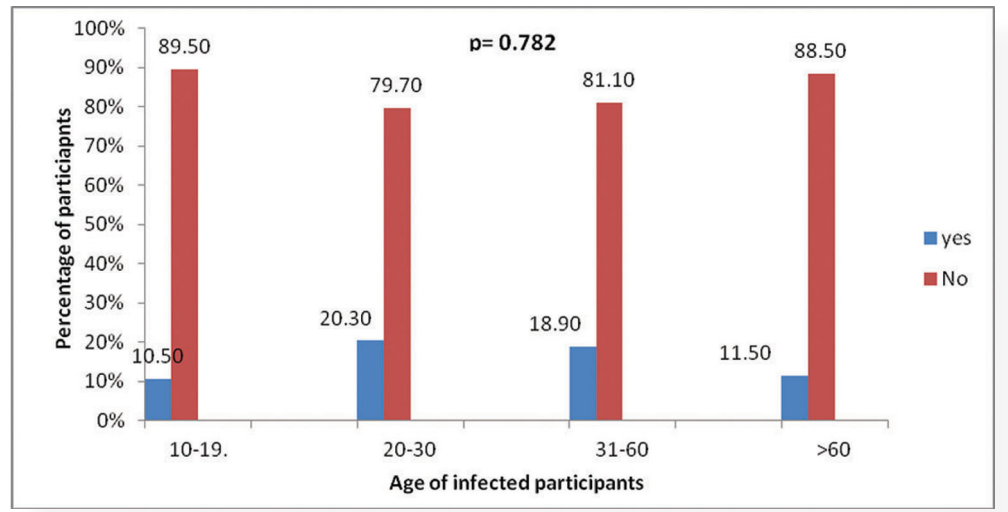

Figure 1. Percentage of infected participants according to age

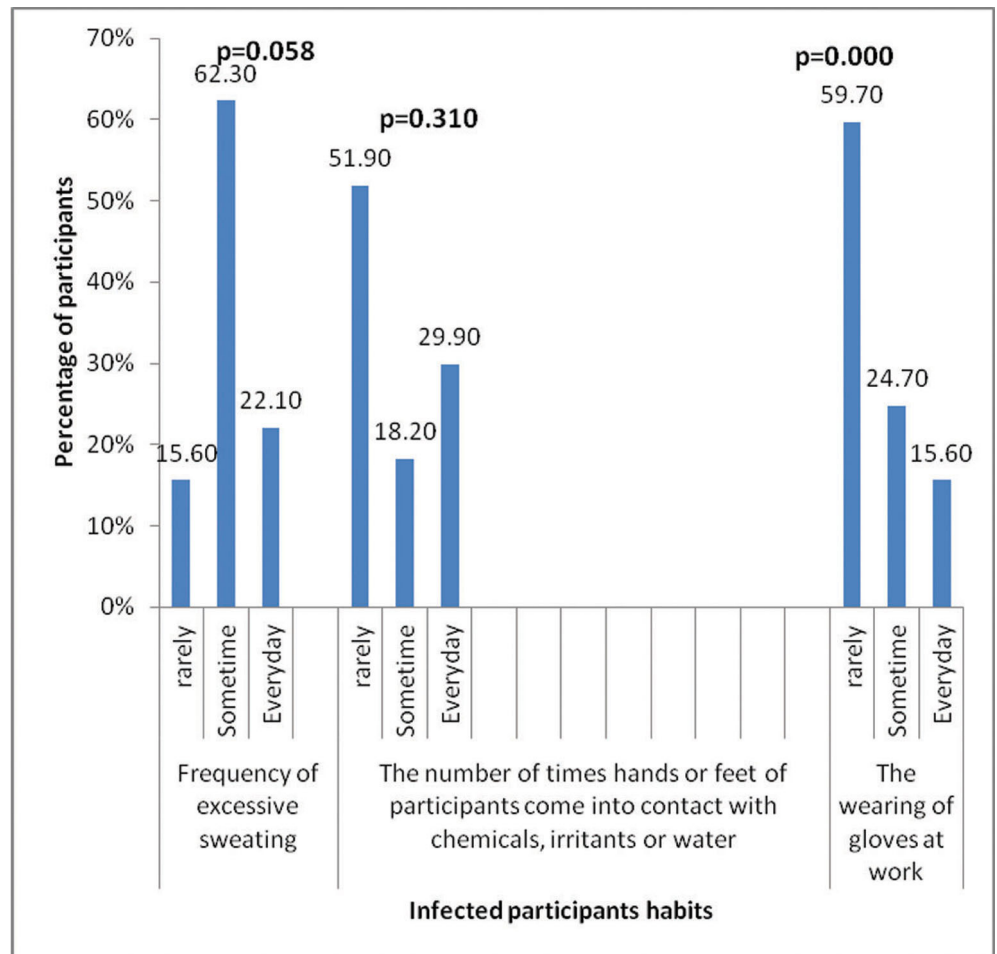

Figure 2. Percentage of infected participants according to their daily habits

\section{Quality of Life (QoL) associated with participants}

$54.5 \%$ of female were unwilling to participate in activities compared to $45.5 \%$ of male. $33.3 \%$ of male were depressed because of their nail infection compared to $66.7 \%$ of female (Fig. 3).

There was a significant correlation observed between nail infection and embarrassment. $(r=0.951 ; p=0.000)$. Same observation was obtained between nail infection and difficulties in doing sports $(\mathrm{r}=0.945 ; \mathrm{p}=0.000)$ as well as problem caused at work or studying $(\mathrm{r}=0.928 ; \mathrm{p}=0.000)$.

More male did not feel embarrassed at all about their nail infection $(11.2 \%)$ while more female $(10.2 \%)$ did not feel any difficulties in doing sport due to their nail infection. $40 \%$ of infected participants did not use any means to hide their infected nails while $34 \%$ used socks to hide their infected nail with a significant difference $(\mathrm{p}=0.000, \mathrm{r}=0.775)$. 


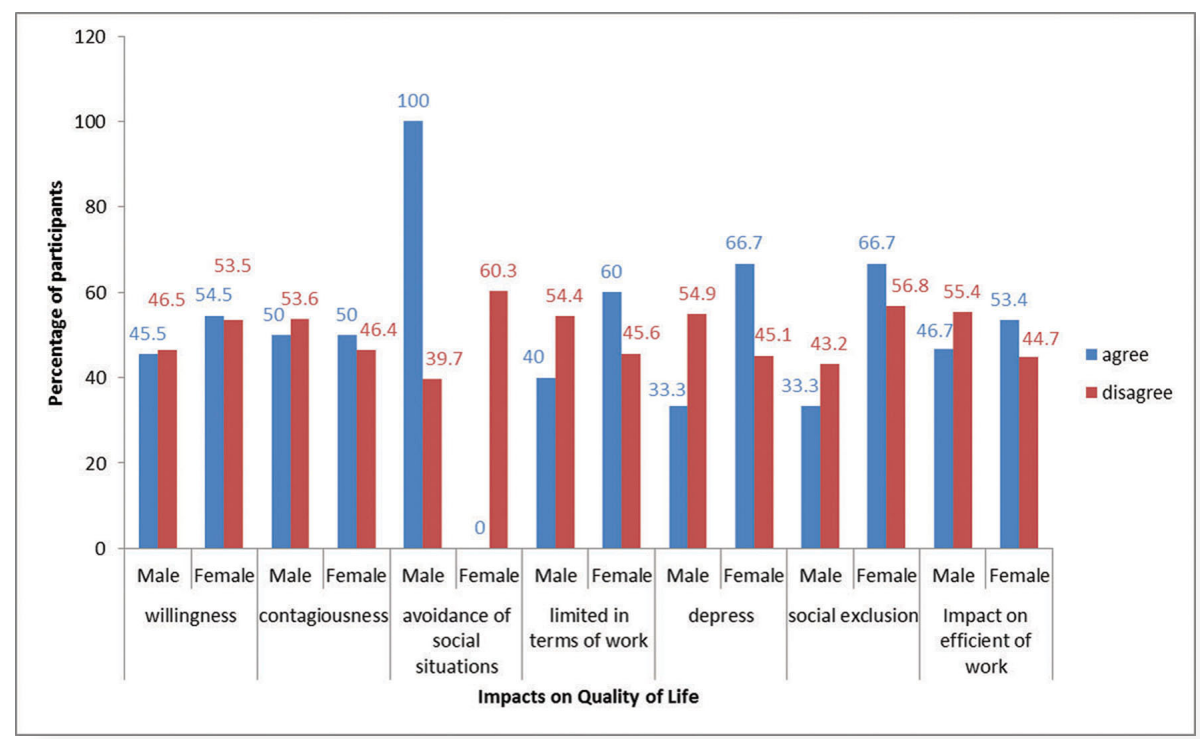

Figure 3. Percentage of infected participants according to Quality of life

\section{Participants' level of awareness}

Figure 4 shows that $68 \%$ of participants responded nail infection as disease. More participants said they consulted a doctor for their nail infection (50.7\%). $54.8 \%$ of them think there is a rapid cure for nail infection. $68.4 \%$ of participants were having treatment for their nail infection while $61.9 \%$ revealed they did not follow the treatment prescribed. $56.1 \%$ of participants were filling to pay for a cure while $71 \%$ of them would accept to follow the treatment even if it is costly. Knowledge on nail infection was higher in male (59.1\%) than in female (40.9\%). $97.2 \%$ of men do not go a manicurist compared to $79.7 \%$ among female. higher percentage of male (74\%) dried their hand and feet after bath compared to $66 \%$ of women. $67.4 \%$ of men and 60.9 of female were aware that water could lead to nail infection. Participants washed their hands, feet and clean their nail more than twice a day while they cut their nail once or more per week. The majority of participants changed their socks once a day while the majority rarely cleaned their nail grooming tools.

Only $20.6 \%$ of participants having primary level of education were aware of how to prevent the spread of nail infection. 53.2\% having done secondary education and $48.2 \%$ having done tertiary level of education were unaware of how to prevent the spread of nail infection. The data was found to be statistically significant $(\mathrm{p}=0.002)$.

\section{Discussion}

Relationship between the severity of nail infection and demographic factors.

The study shows that more than three quarter of the non-diabetic participants did not suffer from any kind of nail infections $(85 \%)$ while $15 \%$ had at least one kind of nail infection. It has been shown that non- diabetic people had a low prevalence of nail infection [14]. Results herein indicate that more male participants $(17.2 \%)$ had nail infections compare to female $(14.1 \%)$ but with similar ratio. However reviews reported that men were three times more prone to nail infection suggesting that this gender difference are not clear but may involve social and/or genetic factors [22].

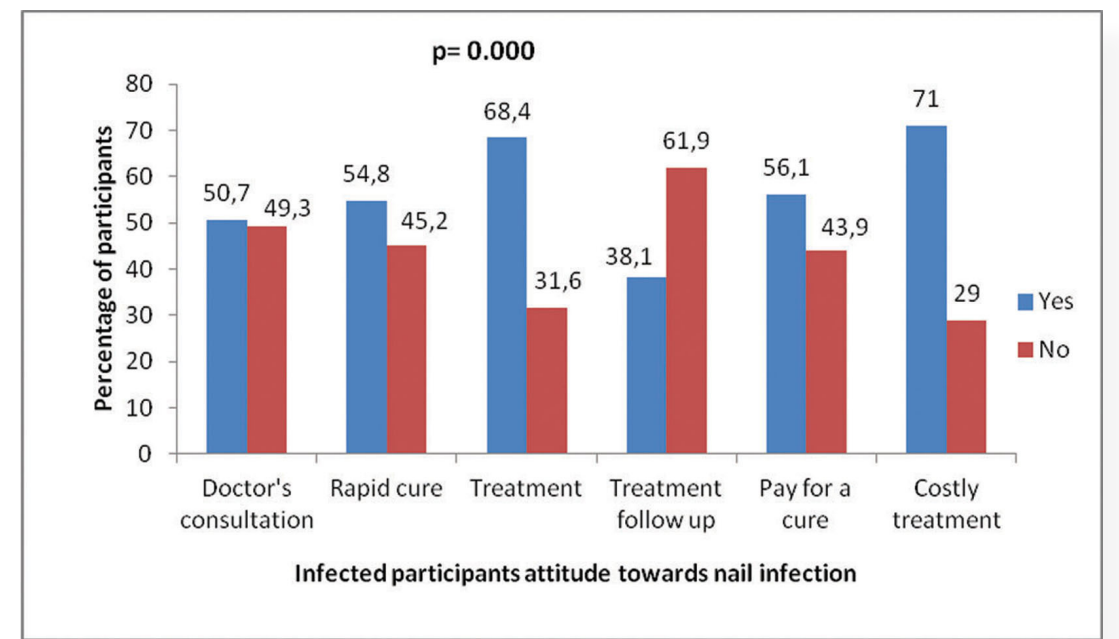

Figure 4. Percentage of participants according to their awareness on nail infection 
As far as age is concerned, it was found that participants within the age group 20-60 years had a higher incidence of nail infection contrary to previous finding which reported that elder citizens were more prone to nail infection. Some of their reasons for age related increase in nail infection include repeated nail trauma, poor peripheral circulation or immunosuppression [15]. Diabetic patients above 66 years old were more prone to nail infection [23].

Significant association is reported between socio economic status of participants and the prevalence of nail infection. The percentage of having nail infection were higher in participants having a total monthly salary of less than US 500 (Rs 15,000) and this is in agreement with the study done previously on nail infection [16,23]. Results indicate that those with higher SES had a lower prevalence of nail infection as hygiene level was good. On the other hand, there was no significant correlation between level of education and frequency of nail infection as it was seen that male having had a tertiary level of education had a higher prevalence of nail infection (44.2\%). However, it was observed that male living in humid regions was more prone to nail infection (43.6\%) compared to $13.2 \%$ of female in humid region. This finding corroborate with previous study which stated that dermatophytes grow well at temperatures of $25-28 \mathrm{oC}$ and infection is more prevalent in warm and humid conditions [24].

Out of the 73 infected participants, it was observed that those who were working had a higher percentage of nail infection for both gender which represents $71.8 \%$ in male and $60.5 \%$ in female. Moreover, it was found that manual workers had more nail infection $(36 \%)$ when compared to other professions like police officer $(28 \%)$ and $21 \%$ for medical professional. As documented in previous study, manual workers are more prone to nail infection due to the fact they are longer exposed to pathogenic fungi at their place of work [25].

\section{Podiatric links to nail infections}

Among the participants, 22\% mentioned that they frequently experienced excessive sweating (hyperhidrosis) in various places of their body. A more profound analysis concluded that infected participant primary experienced face hyperhydrosis $(37.1 \%)$ while $22.3 \%$ had hyperhydrosis to their feet. Furthermore, among those subjects who had nail infection, it was found that a significant correlation exists between sweating and the approximate number of hours participants wear shoes per day, implying that prolonged wearing of shoes resulted in nail infection. These findings correspond with previous study which reported that hyperhidrosis provided a conducive environment for proliferation of dermatophytes [25]. It was observed that there was no correlation between contacts with chemical or water and prevalence of nail infection. However, the frequency of wearing gloves at work could be linked with the prevalence of nail infection. The positive correlation implies that as the frequency of wearing gloves increases, the risk of getting nail infection increases too.

It was observed that more male experienced hardening of the nail $(5.1 \%)$ while $5.1 \%$ female had a greater incidence of nail discoloration. Furthermore it was found that more male had multiple signs of nail infection brittleness, discoloration and hardening amongst others which represent $2.8 \%$ of the $17.2 \%$ male infected. The prevalence of infecting fingernail was low in both male and female representing $9.3 \%$ and $8.2 \%$ respectively while it was observed that both male and female were more prone of to toenail infection. A possible reason could be fungal nail infections of the toenail have more time to grow and spread, because toenails grow more slowly than fingernails [27].

Data obtained in this study indicates a positive correlation between nail infection and other associated medical problems. When family history was assessed, it was found that $45 \%$ of infected participants had member of their family who had diabetes while $26 \%$ reported having nail, hair or skin problems in their family. Previous studies report that genetic factors play a fundamental role in nail infection as affected children had at least one parent with Distal Subungual Onychomycosis (DSO) [28].

This study also reports that all studied patients with nail infections experienced at least one of the following sensations that are tingling, burning and numbness. It was also observed that $12.8 \%$ male and $31.6 \%$ female had difficulties in cutting their infected nails. Furthermore, $7.7 \%$ male and $18.4 \%$ female had problem while wearing shoes due to their nail infections. This finding support previous research done on podiatric difficulties of infected participants [26].

\section{Association of embarrassment and discomfort of nail infection and participant's Quality of Life (QoL)}

Researchers have found that nail infection significantly decreases the Quality of Life (QoL) of patients [18]. A similar scenario is depicted here where participants $(45.5 \%$ male and $54.5 \%$ female) agreed that they were unwilling to participate in activities due to their nail infection. Male suffering from any kind of nail infection avoided social situations more than women. On the other hand, more female $(60 \%)$ reported that they were limited in terms of work when compared to male (40\%). This finding goes in line with what was found in India where it was reported that patients having nail infection faced limitation of physical activities thus making it impossible to perform simple tasks of daily life [29]. Same trend applies to the female gender where depression is concerned. $66.7 \%$ of female participants were depressed due to their nail infection compared to only $33.3 \%$ of male participants. Higher number of women $(66.7 \%)$ feel that they were socially excluded due to their nail infection than men $(33.3 \%) .53 .4 \%$ of female participants felt that their nail infection had a significant impact on their efficiency at work while only $46.7 \%$ felt so. It was also observed that nail infection impaired the QoL of women more consequently than those for men. However no statistical significance was noted between age and the QoL of the participants and level of education and the QoL of the participants contrary to what were found in a previous study [18].

A major finding of this study was that nail infection was more likely to cause embarrassment in women $(6 \%)$ than in men $(3.9 \%)$. On the other hand, men experienced more difficulties in doing sport activities $(3.7 \%)$ due to their nail infection compared to women which was just $0.8 \%$. The prevalence of nail infection also had a greater impact on female's work and in studies than the opposite gender. These results are consistent with those obtained in Poland and India by respectively [18,29]. $34 \%$ of infected participants admitted that they hide their nail infection using socks while $23 \%$ of them used closed shoes and this was statistically significant. The present study also demonstrates a positive correlation among the participants between nail infection and type of shoes participants wears which implies that the types of shoes worn by participants could lead to nail infection. It was reported that only $21.4 \%$ of diabetic having nail infection wore medicated shoes as they did not know about the importance of the medicated shoes or they stated that medicated shoes are too expensive [23]. 
Level of awareness of participants towards nail infection

The current study found that $68 \%$ of participants viewed nail infection as a disease while $12 \%$ think it is an aesthetic problem. This contradicts past studies which consider nail infection as a serious medical problem [18]. It was also found that among nail infected participants $(\mathrm{n}=73), 50.7 \%$ visit a doctor for their nail infection and out of this, $68.4 \%$ of them were treating the infected nail with prescribed medicine. 45 out of the 73 participants having nail infection revealed neglecting their treatment follow-up suggesting that these participants were unaware of the consequence of the nail infection if left untreated over a long period which may lead to complications ranging from gangrene to amputation [30]. Interestingly, 56.1\% of the subject would be willing to pay for a cure while $71 \%$ of participants were still willing to pay for a cure even if the treatment would be costly showing that cost was not a factor from preventing them seeking medical help. These finding were found to be statistically significant and goes in line with other studies [9]. Negligence however is not uncommon as $68.4 \%$ of diabetic patients having nail infection did not treat their nails while $29.1 \%$ never went to the podiatrist at all [23].

As far as knowledge is concerned, it is observed that male was more aware of nail infection $(59.1 \%$ in men and $40.9 \%$ in female) but most infected participants did not know how they acquired nail infection (35.9\% in men and $65.8 \%$ in women). Male were more delicate in drying their feet or hand after a bath $(74 \%)$ compared to $26 \%$ of women. Hygienic conditions like cutting, cleaning of nails and washing of feet and hands regularly were well known among the participants. However it was observed that a higher incidence of participants rarely or never cleaned their nail cutter before using it (not statistically significant). This finding shows how participants neglect the fact that fungi on nail cutter could contaminate healthy nails. Most participants were aware of the importance of doing a check-up by a podiatrist, to cut nail once a week and to avoid nail injuries amongst others but the results were not found to be statistically significant. However, irrespective of the education level and age, it was found that more than half of participants did not know about the routes of nail infections or the precautions needed to avoid further contamination of healthy nails. This suggests that even mature or well literate participants had little knowledge of the proliferation of nail infections.

\section{Conclusion}

In the study nail infection were observed to rarely affect participants below 19 years old but had a greater prevalence in adults of age 20 to 50. Prevalence rate is higher in male. Only $15 \%$ of non-diabetic participants had nail infection showing a low prevalence among them. Occupation could be a risk factor for nail infection with manual workers more susceptible. QoL has been found to be impaired in all domains ranging from physical, mental to social functioning. Thus nail infection must be considered as a serious medical problem owing careful consideration from podiatrist. Female were more embarrassed compared to male while more male avoided social situations than female. Toenails were more prone to nail infections than fingernail. Many of the participants were not aware of the existing problem on nail infection even though they show signs and symptoms and majority were unaware of the mode of transmission or means of preventing infection.

\section{Acknowledgements}

I wish to acknowledge Dr. H. Doorgakant, Podiatrist at the Victoria Hospital as well as the medical staff of Medical Unit of Victoria Hospital for their collaboration in data collection. I am most grateful to my colleague Mr. Ganesh Sujeewon for helping me throughout this study and my friends for their immense help. The support of the Department of Health Sciences, Faculty of Science, University of Mauritius is also acknowledged.

\section{REFERENCES}

1. Bonifaz A, Vázquez-González D, Saúl A, Fierro-Arias L, PonceOlivera MR: Refractory onychomycosis due to trichophyton rubrum: combination therapy with itraconazole and terbinafine. Our Dermatol Online. 2011;3,108-12.

2. Dhavalshankh G, Dhavalshankh A, Masvekar V: Cutaneous manifestations of dermatomyositis in male patient: a rare report. Our Dermatol Online. 2013;4,168-71.

3. Chang P: Dorsal ungual pterygium. Our Dermatol Online. 2012;3,57-60.

4. Sikora M, Pacholek T, Soter K, Szepietowski J: Analysis of fungal skin and skin appendages infections in the region of Wroclaw in the years 1995-1999. Mikol Lek. 2000;7:145-51.

5. Kaur T, Puri N: Onychomycosis - a clinical and mycological study of 75 cases. Our Dermatol Online. 2012;3:172-7.

6. Puri N, Kaur T: A study of nail changes in various dermatosis in Punjab, India. Our Dermatology Online. 2012;3:164-70.

7. Drake LA, Patrick D, Fleckman P: The impact of onychomycosis on quality of life: development of an international onychomycosisspecific questionnaire to measure patient quality of life. J Am Acad Dermatol. 1999;41:189-96.

8. Havu V, Heikkila H, Kuokkanen K: A double-blind, randomized study to compare the efficacy and safety of terbinafine with fluconazole in the treatment of onychomycosis. Br J Dermatol. 2000;142:97-102.

9. Drake LA, Scher RK, Smith EB: Effect of onychomycosis on quality of life. J Am Acad Dermatol. 1998;38:702-4.

10. Scher RK: Onychomycosis: therapeutic update. J Am Acad Dermatol. 1999;40:21-6.

11. Elewski B, Charif M, Daniel C. 3rd: Onychomycosis. in: Scher RK, Daniel C III, editors. Nails: diagnosis, treatment, surgery, 2nd ed. Philadelphia: WB Saunders. 1997;151-162.

12. Kaur R, Kashyap B, Bhalla P: Onychomycosis-epidemiology, diagnosis and management. Indian J Med Microbiol. 2008;26:10816.

13. Mahreen A: Epidemiology of superficial fungal infections. Clin Dermatol. 2010;28:197-201

14. Pierard GP, Pierard-Franchimont GP: The nail under fungal siege in patients with type II Diabetes Mellitus. Mycoses. 2005;48:339-42. 15. Gupta AK, Jain HC, Lynde CW, Macdonald P, Cooper EA, Summerbell RC: Prevalence and epidemiology of onychomycosis in patients visiting physicians' offices: a multicenter Canadian survey of 15,000 patients. J Am Acad Dermatol. 2000;43:244-8.

16. Hapcioglu B, Yegenoglu Y, Disci R, Erturan Z and Kaymakcalan H: Epidemiology of Superficial Mycosis (Tinea Pedis, Onychomycosis) in Elementary School Children in Istanbul, Turkey. Coll Antropol. 2006;30:119-24.

17. Nail repair, 2013. Too embarrassed to seek treatment for nail infections. [online]

18. Szepietowski JC, Reich A: Onychomycosis and quality of life. Europ Dermatol. 2009;1:85-6.

19. Sujeewon G: A study on onychomycosis in a random sample of diabetic patients. Thesis (BSc). 2006. University of Mauritius. 
20. Ramana Y: Survey of onychomycosis among agricultural workers. Thesis (Diploma), 2005. University of Mauritius.

21. Finlay A.Y, Khan G.K: Dermatology life quality index. April 1992.

22. Gupta AK, Konnikov N, MacDonald P, Rich P, Rodger NW, Edmonds MW et al: Prevalence and epidemiology of toenail onychomycosis in diabetic subjects: a multicentre survey. $\mathrm{Br} \mathrm{J}$ Dermatol. 1998;139:665-71.

23. Chooneea M, Jeewon R: A survey of nail infection and awareness among diabetic patients in Mauritius. 2013 (unpublished article).

24. Blanka H, Czaika A, Friedrich M: Epidemiological trends in skin mycoses worldwide. Mycoses. 2008;51:2-15.

25. Urbanowski S, Kosmowski W, Gwiezdzinski Z, Araszkiewicz A: Quality of life and depression among patients with onychomycosis. Clin Dermatol. 2000: Singapore: 1998.
26. Drake LA, Dinehart SM, Farmer ER, Goltz RW, Graham GF, Hordinsky MK et al: Guidelines of care for superficial mycotic infections of the skin: onychomycosis. J Am Acad Dermatol. 1996,34:116-21.

27. Haneke E: Nail psoriasis, psoriasis, dr. jennifer soung (ed.), 2012. intech.

28. Zaias N, Tosti A, Rebell G, Morelli R, Bardazzi F, Bieley H et al :Autosomal dominant pattern of distal subungual onychomycosis caused by Trichophyton rubrum. Am Acad Dermatol. 1996;34:3024.

29. Singal A, Khanna D: Onychomycosis: Diagnosis and management. Indian J Dermatol Venereol Leprol. 2011;77:659-72.

30. Frykberg RG, Armstrong DG, Giurini J, Edwards SA, Kravette M, Kravitz S et al: Diabetic foot disorders: a clinical practice guideline. Journal Foot Ankle Surg. 2000;39:1-60. permits unrestricted use, distribution, and reproduction in any medium, provided the original author and source are credited. 